\title{
A separate lowstand lake at the northern edge of Lake Tanganyika? Evidence from phylogeographic patterns in the cichlid genus Tropheus
}

\author{
Christian Sturmbauer (D) Christine Börger • \\ Maarten Van Steenberge • Stephan Koblmüller
}

Received: 24 February 2016/Revised: 8 July 2016/Accepted: 31 July 2016/Published online: 19 August 2016

(C) The Author(s) 2016. This article is published with open access at Springerlink.com

\begin{abstract}
In Lake Tanganyika, lake level fluctuations were shown to have had a major impact on the evolution of littoral species. Many species are subdivided into arrays of populations, geographical races and sister species, each colonizing a particular section of the shore. Their often limited dispersal abilities promoted geographic isolation and, on the long run, allopatric speciation. With more than 120 distinct populations, the genus Tropheus represents the most spectacular and best-studied example of this phenomenon. The present study aims at the fine-scale reconstruction of the spread of two mitochondrial Tropheus-lineages in the very north of the lake, where two species, T. sp. 'black' and T. brichardi, occur. Using mtDNA sequences and AFLP-data, we ana-
\end{abstract}

Electronic supplementary material The online version of this article (doi:10.1007/s10750-016-2939-8) contains supplementary material, which is available to authorized users.

Guest editors: S. Koblmüller, R. C. Albertson, M. J. Genner, K. M. Sefc \& T. Takahashi / Advances in Cichlid Research II: Behavior, Ecology and Evolutionary Biology

C. Sturmbauer $(\bowtie) \cdot$ C. Börger · M. Van Steenberge ·

S. Koblmüller

Department of Zoology, University of Graz,

Universitätsplatz 2, $8010 \mathrm{Graz}$, Austria

e-mail: christian.sturmbauer@uni-graz.at

M. Van Steenberge

Department of Ichthyology, Royal Museum for Central Africa, Leuvensesteenweg 13, 3080 Tervuren, Belgium lyzed samples from 21 localities and found a highly complex conglomerate of introgressed populations formed by the repeated contact of two lineages. Our data suggest repeated cross-lake dispersal of $T$. sp. 'black' haplotypes along the ridge between the West and East Ubwari Fault, supporting an additional persisting lowstand-lake in the Bujumbura subbasin at the very north of the lake and highlighting once more the impact of lake level fluctuations on the genetic structure and evolution of stenotopic rockdwelling cichlid species.

Keywords mtDNA sequences - Control region AFLP · Secondary admixis · Hybridization · Lake level fluctuations

\section{Introduction}

Genetic and phenotypic divergence among populations forms the basis of speciation events. However,

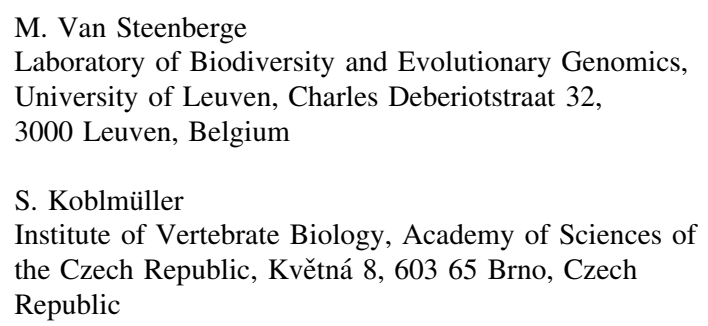


such pathways rarely proceed in a linear fashion by divergence alone, as repeated intermezzos of gene flow perturbate gene pools and set the stage for selection, drift, and speciation (King \& Lawson, 1995; Rossiter, 1995; Pinho \& Hey, 2010; Sturmbauer, 2011). On the long run, secondary admixis can either render population distinctness or produce novel and distinct hybrid entities, which can evolve to novel species (Seehausen, 2004; Nolte \& Tautz, 2010).

Following the spatial segregation into rock- and sand-habitats along the shores of Lake Tanganyika, many stenotopic littoral species are subdivided into distinct populations that vary mainly in coloration. The genus Tropheus represents perhaps the best example for this phenomenon. Tropheus is abundant in the upper littoral zone in all types of rocky habitats, where it feeds on epilithic algae and takes shelter from predators. Sandy or muddy shores and river estuaries are strictly avoided, resulting in about 120 distinct local variants, some of which live in sympatry (Schupke, 2003; Konings, 2013). The six described species (Poll, 1986) are currently under revision (Van Steenberge, 2014).

A series of previous studies on the evolution and colonization history of this genus set out to reconstruct the origin and spread of this highly specialized rockdwelling species-complex (Sturmbauer \& Meyer, 1992; Sturmbauer et al., 1997; Rüber et al., 1999; Baric et al., 2003; Sturmbauer et al., 2005; Egger et al., 2007; Sefc et al., 2007, 2016; Koblmüller et al., 2011; Nevado et al., 2013). These studies suggested mitochondrial introgression, sometimes on a small scale between presently allopatric populations, sometimes on a large-scale producing true hybrid populations, at various time points in the evolutionary history of the genus Tropheus. Most of these events of population displacement were triggered by lake level fluctuations, which extended to a few hundred meters below present level (Lezzar et al., 1996; Cohen et al., 1997; Scholz et al., 2003; McGlue et al., 2008). While the effect is widely seen in population genetic studies on various Lake Tanganyika cichlid species (Verheyen et al., 1996; Rüber et al., 1999, 2001; Duftner et al., 2006; Koblmüller et al., 2007, 2009, 2011; Sefc et al., 2007; Nevado et al., 2013; Van Steenberge et al., 2015), studies specifically testing for the impact of Pleistocene water level fluctuations are scarce (Koblmüller et al., 2011; Sefc et al., 2016; Winkelmann et al., 2016).
In the present study, we aim at the fine-scale reconstruction of the origin and spread of the-in terms of colors-highly divergent Tropheus populations in the very north of Lake Tanganyika. In this part of the Lake, three species of Tropheus are found. Besides the basal species T. duboisi Marlier 1959, these are T. brichardi Nelissen \& Thys van den Audenaerde 1975 and the hitherto undescribed species $T$. sp. 'black' (Konings, 2013). Tropheus duboisi co-occurs with $T$. sp. 'black' at Bemba, and with T. brichardi at Mwamugongo. At the eastern shore of the Ubwari Penninsula between Cape Caramba and Muzimu two Tropheus live in sympatry, one currently assigned to $T$. sp. 'black' named Caramba, and the second tentatively assigned to $T$. cf. brichardi named Ubwari-green in the aquarium trade (Schupke, 2003; Konings, 2013). We analyzed samples from 21 populations of $T$. brichardi and $T$. sp. 'black' and found a highly complex conglomerate of populations, formed by the repeated contact of two ancient mtDNA lineages, and relate the findings to patterns in nuclear DNA markers using new AFLP data.

\section{Materials and methods}

Samples and molecular techniques

This study is based on 179 sequences of the most variable part of the mitochondrial control regions and AFLP profiles of 30 individuals. Fin clips of Tropheus brichardi and $T$. sp. 'black' were collected from 21 localities in the northernmost section of Lake Tanganyika (Fig. 1) during several expeditions between 1991 and 2013 (Van Steenberge, 2011), or obtained via the aquarium trade, and preserved in $>96 \%$ ethanol. Mitochondrial control region sequences for 27 of these samples have been published previously (Sturmbauer \& Meyer, 1992; Baric et al., 2003; Sturmbauer et al., 2005; Egger et al., 2007), and 152 new samples were sequenced in this study (Table 1). Whole genomic DNA was extracted following a rapid Chelex protocol (Richlen \& Barber, 2005) or using the Macherey-Nagel Nucleospin extraction kit, following the manufacturer's instructions.

For all 152 new samples, the most variable part of the mitochondrial control region was amplified and sequenced following Koblmüller et al. (2011) and Duftner et al. (2005), respectively. The primers used for PCR amplification and chain termination sequencing 
Fig. 1 Map of northern Lake Tanganyika with sampling sites. Numbers in parentheses refer to sample size; pie charts indicate assignment to major haplogroups (Fig. 2), with size of the pie chart proportional to the number of samples. Square symbols marking sampling sites indicate the occurrence of $T$. brichardi instead of $T$. sp. 'black' (circle symbols). The triangle symbol denotes the occurrence of $T$. cf. brichardi named Ubwarigreen in the aquarium trade

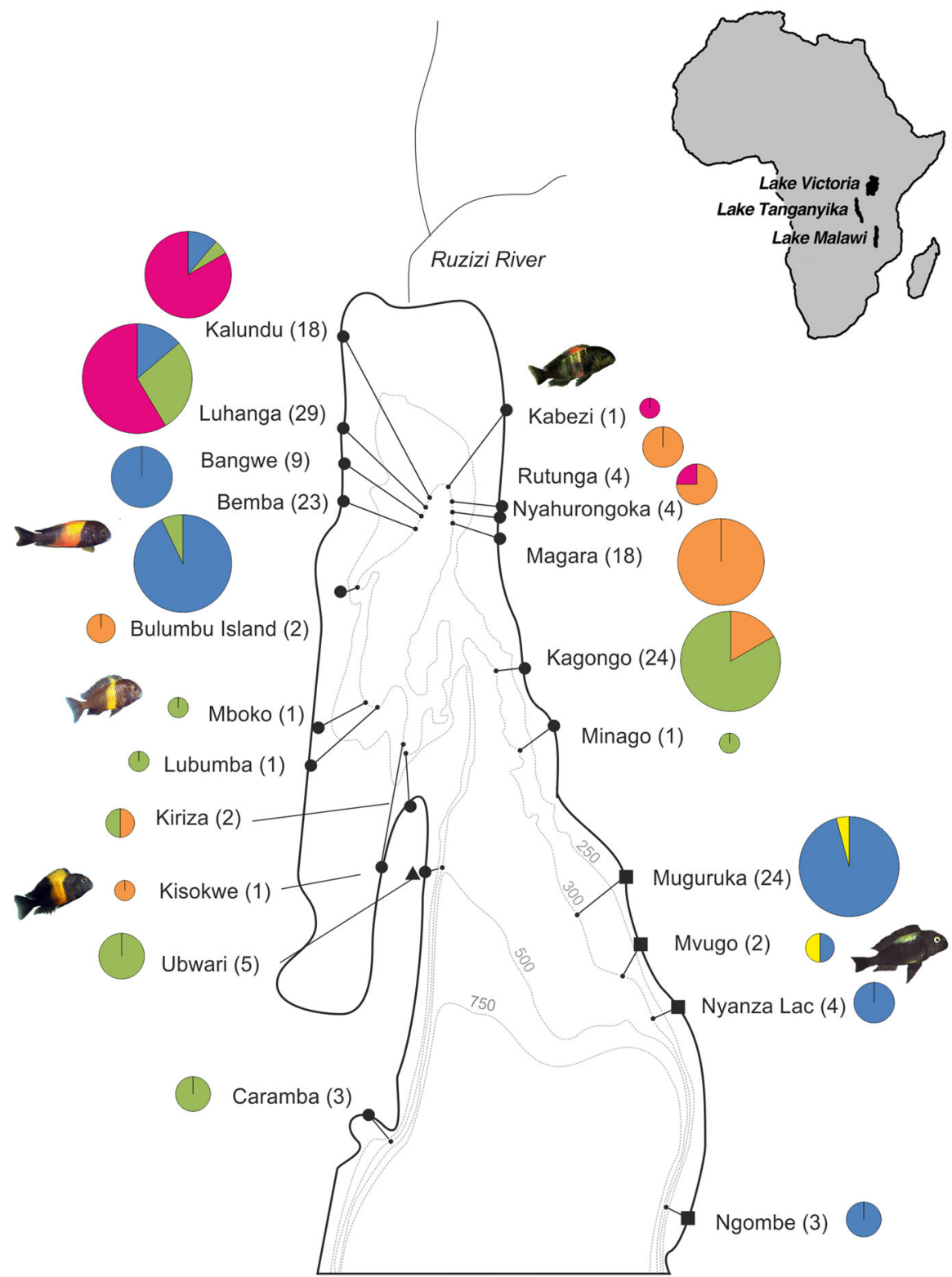

were L-Pro-F (Meyer et al., 1994) and TDK-D (Lee et al., 1995). DNA fragments were purified with SephadexTM G-50 (GE Healthcare) and visualized on an ABI 3130xl automated sequencer (Applied Biosystems). Sequences were aligned using ClustalW in the computer program MEGA V6.0 (Tamura et al., 2013) and the resulting alignment was controlled by eye to check for obvious alignment errors. The final alignment length was $352 \mathrm{bp}$. The new DNA-sequences have been deposited in Genbank. Sample information and all accession numbers are listed in Table 1.
AFLP genotyping of 30 individuals (ten primer combinations for selective amplification: EcoRI-ACA/ $M s e$-CAA, EcoRI-ACT/MseI-CAG, EcoRI-ACC/MseICAC, EcoRI-ACA/MseI-CAG, EcoRI-ACA/MseI-CAC, EcoRI-ACA/MseI-CAT, EcoRI-ACT/MseI-CAT, Eco $R \mathrm{I}-\mathrm{ACT} / M s e \mathrm{I}-\mathrm{CAA}, \quad E c o R \mathrm{I}-\mathrm{ACT} / M s e \mathrm{I}-\mathrm{CAC}, E c o R \mathrm{I}-$ ACC/MseI-CAA) followed the protocol described in Egger et al. (2007). Selective PCR products were sized against an internal standard (GeneScan-500 ROX, Applied Biosystems) on an ABI 3130xl automated sequencer (Appied Biosystems). The AFLP data are part 
Table 1 Characterization of the individuals studied, with information concerning sampling locations and their GPS coordinates, extraction number, GenBank accession number, species and haplotypes to which individuals were assigned as well as the sample identification

\begin{tabular}{|c|c|c|c|c|c|c|c|}
\hline Location & Latitude (S) & Longitude (E) & Extraction no. & Accession no. & Species & Haplotype & Sample ID \\
\hline \multirow[t]{9}{*}{ Bangwe } & \multirow[t]{9}{*}{$-3,7919$} & \multirow[t]{9}{*}{29,1627} & 14420 & KX513704 & T. sp. 'black' & Ht_01 & $\mathrm{KS} 23 \mathrm{H} 4$ \\
\hline & & & 14423 & KX513701 & T. sp. 'black' & Ht_02 & $\mathrm{KS} 23 \mathrm{H} 4$ \\
\hline & & & 14424 & KX513700 & T. sp. 'black' & Ht_02 & KS23H5 \\
\hline & & & 14454 & KX513671 & T. sp. 'black' & Ht_02 & T14/03/A4 \\
\hline & & & 14455 & KX513670 & T. sp. 'black' & Ht_02 & T14/03/A5 \\
\hline & & & 14419 & KX513705 & T. sp. 'black' & Ht_03 & $\mathrm{KS} 23 \mathrm{H} 4$ \\
\hline & & & 14421 & KX513703 & T. sp. 'black' & Ht_03 & $\mathrm{KS} 23 \mathrm{H} 4$ \\
\hline & & & 14422 & KX513702 & T. sp. 'black' & Ht_03 & $\mathrm{KS} 23 \mathrm{H} 4$ \\
\hline & & & 14426 & KX513699 & T. sp. 'black' & Ht_03 & KS23H5 \\
\hline \multirow[t]{23}{*}{ Bemba } & \multirow[t]{23}{*}{$-3,7919$} & \multirow[t]{23}{*}{29,1627} & & Z12099 & T. sp. 'black' & Ht_01 & \\
\hline & & & & Z12091 & T. sp. 'black' & Ht_01 & \\
\hline & & & & Z12100 & T. sp. 'black' & Ht_01 & \\
\hline & & & & Z12098 & $T$. sp. 'black' & Ht_01 & \\
\hline & & & & Z72097 & T. sp. 'black' & Ht_01 & \\
\hline & & & & Z12095 & T. sp. 'black' & Ht_01 & \\
\hline & & & & Z12093 & T. sp. 'black' & Ht_01 & \\
\hline & & & & Z12092 & T. sp. 'black' & Ht_01 & \\
\hline & & & 14451 & KX513674 & T. sp. 'black' & Ht_01 & T14/03/A1 \\
\hline & & & 14388 & KX513736 & T. sp. 'black' & Ht_01 & KS22J6 \\
\hline & & & 14389 & KX513735 & T. sp. 'black' & Ht_01 & KS22J6 \\
\hline & & & 14393 & KX513731 & T. sp. 'black' & Ht_01 & KS23A10 \\
\hline & & & 14394 & KX513730 & T. sp. 'black' & Ht_01 & KS23A10 \\
\hline & & & 14390 & KX513734 & T. sp. 'black' & Ht_02 & KS22J6 \\
\hline & & & 14391 & KX513733 & T. sp. 'black' & Ht_03 & KS22J6 \\
\hline & & & 14392 & KX513732 & T. sp. 'black' & Ht_04 & KS23A10 \\
\hline & & & 14452 & KX513673 & T. sp. 'black' & Ht_25 & T14/03/A2 \\
\hline & & & 14453 & KX513672 & T. sp. 'black' & Ht_26 & T14/03/A3 \\
\hline & & & & Z12096 & T. sp. 'black' & Ht_03 & \\
\hline & & & & Z12094 & T. sp. 'black' & Ht_03 & \\
\hline & & & 14387 & KX513737 & T. sp. 'black' & Ht_01* & KS22J6 \\
\hline & & & 816 & KX513597 & T. sp. 'black' & Ht_02* & 816 \\
\hline & & & 820 & KX513596 & T. sp. 'black' & Ht_02* & 820 \\
\hline \multirow[t]{2}{*}{ Bulumbu } & \multirow[t]{2}{*}{$-3,77$} & \multirow[t]{2}{*}{29,12} & 833 & KX513604 & T. sp. 'black' & Ht_51 & 833 \\
\hline & & & 835 & KX513595 & T. sp. 'black' & Ht_15* & 835 \\
\hline \multirow[t]{3}{*}{ Caramba } & \multirow[t]{3}{*}{$-4,5$} & \multirow[t]{3}{*}{29,18} & 14965 & KX513738 & T. sp. 'black' & Ht_59 & T14/05/A6 \\
\hline & & & & Z75702 & T. sp. 'black' & Ht_48 & \\
\hline & & & 14966 & KX513739 & T. sp. 'black' & Ht_60* & T14/05/A7 \\
\hline Kabezi & $-3,5$ & 29,333 & & Z75694 & T. sp. 'black' & Ht_47 & \\
\hline \multirow[t]{6}{*}{ Kagongo } & \multirow[t]{6}{*}{$-3,7352$} & \multirow[t]{6}{*}{29,5847} & 14415 & KX513709 & T. sp. 'black' & Ht_15 & KS20I9 \\
\hline & & & 14502 & KX513624 & T. sp. 'black' & Ht_15 & $\mathrm{T} 14 / 03 / \mathrm{F} 2$ \\
\hline & & & 14416 & KX513708 & T. sp. 'black' & Ht_16 & KS20I9 \\
\hline & & & 14427 & KX513698 & T. sp. 'black' & Ht_16 & KS20I5 \\
\hline & & & 14432 & KX513693 & T. sp. 'black' & Ht_16 & KS20I6 \\
\hline & & & 14495 & KX513631 & T. sp. 'black' & Ht_16 & $\mathrm{T} 14 / 03 / \mathrm{E} 5$ \\
\hline
\end{tabular}


Table 1 continued

\begin{tabular}{|c|c|c|c|c|c|c|c|}
\hline Location & Latitude (S) & Longitude (E) & Extraction no. & Accession no. & Species & Haplotype & Sample ID \\
\hline & & & 14498 & KX513628 & T. sp. 'black' & Ht_16 & T14/03/E8 \\
\hline & & & 14418 & KX513706 & T. sp. 'black' & Ht_17 & KS20I9 \\
\hline & & & 14496 & KX513630 & T. sp. 'black' & Ht_17 & T14/03/E6 \\
\hline & & & 14501 & KX513625 & T. sp. 'black' & Ht_17 & $\mathrm{T} 14 / 03 / \mathrm{F} 1$ \\
\hline & & & 14503 & KX513623 & T. sp. 'black' & Ht_17 & $\mathrm{T} 14 / 03 / \mathrm{F} 3$ \\
\hline & & & 14431 & KX513694 & T. sp. 'black' & Ht_18 & KS20I5 \\
\hline & & & 14434 & KX513691 & T. sp. 'black' & Ht_19 & KS20I6 \\
\hline & & & 14497 & KX513629 & T. sp. 'black' & Ht_40 & T14/03/E7 \\
\hline & & & 14417 & KX513707 & T. sp. 'black' & Ht_16 & KS20I9 \\
\hline & & & 14428 & KX513697 & T. sp. 'black' & Ht_16 & KS20I5 \\
\hline & & & 14429 & KX513696 & T. sp. 'black' & Ht_16 & KS20I5 \\
\hline & & & 14430 & KX513695 & T. sp. 'black' & Ht_16 & KS20I5 \\
\hline & & & 14433 & KX513692 & T. sp. 'black' & Ht_16 & KS20I6 \\
\hline & & & 14494 & KX513632 & T. sp. 'black' & Ht_15 & $\mathrm{T} 14 / 03 / \mathrm{E} 4$ \\
\hline & & & 14499 & KX513627 & T. sp. 'black' & Ht_16 & T14/03/E9 \\
\hline & & & 14500 & KX513626 & T. sp. 'black' & Ht_16 & $\mathrm{T} 14 / 03 / \mathrm{E} 10$ \\
\hline & & & 14493 & KX513633 & T. sp. 'black' & Ht_15* & T14/03/E3 \\
\hline & & & 14492 & KX513634 & T. sp. 'black' & Ht_16* & T14/03/E2 \\
\hline \multirow[t]{18}{*}{ Kalundu } & $-3,388$ & 29,144 & 14438 & KX513687 & T. sp. 'black' & Ht_01 & KS24G3 \\
\hline & & & 14476 & KX513650 & T. sp. 'black' & Ht_10 & T14/03/C6 \\
\hline & & & 14436 & KX513689 & T. sp. 'black' & Ht_11 & KS24G3 \\
\hline & & & 14439 & KX513686 & T. sp. 'black' & Ht_11 & KS24G3 \\
\hline & & & 14473 & KX513653 & T. sp. 'black' & Ht_11 & $\mathrm{T} 14 / 03 / \mathrm{C} 3$ \\
\hline & & & 14478 & KX513648 & T. sp. 'black' & Ht_11 & $\mathrm{T} 14 / 03 / \mathrm{C} 8$ \\
\hline & & & 14481 & KX513645 & T. sp. 'black' & Ht_11 & $\mathrm{T} 14 / 03 / \mathrm{D} 1$ \\
\hline & & & 14441 & KX513684 & T. sp. 'black' & Ht_14 & KS24G4 \\
\hline & & & 14474 & KX513652 & T. sp. 'black' & Ht_14 & $\mathrm{T} 14 / 03 / \mathrm{C} 4$ \\
\hline & & & 14437 & KX513688 & T. sp. 'black' & Ht_20 & KS24G3 \\
\hline & & & 14442 & KX513683 & T. sp. 'black' & Ht_21 & KS24G4 \\
\hline & & & 14475 & KX513651 & T. sp. 'black' & Ht_21 & T14/03/C5 \\
\hline & & & 14472 & KX513654 & T. sp. 'black' & Ht_30 & $\mathrm{T} 14 / 03 / \mathrm{C} 2$ \\
\hline & & & 14479 & KX513647 & T. sp. 'black' & Ht_35 & T14/03/C9 \\
\hline & & & 14477 & KX513649 & T. sp. 'black' & Ht_38 & $\mathrm{T} 14 / 03 / \mathrm{C} 7$ \\
\hline & & & 14480 & KX513646 & T. sp. 'black' & Ht_35 & $\mathrm{T} 14 / 03 / \mathrm{C} 10$ \\
\hline & & & 14435 & KX513690 & T. sp. 'black' & Ht_01* & KS24G3 \\
\hline & & & 14440 & KX513685 & T. sp. 'black' & Ht_11* & KS24G4 \\
\hline \multirow[t]{2}{*}{ Kiriza } & $-4,05$ & 29,216 & & $\mathrm{Z75700}$ & T. sp. 'black' & Ht_16 & \\
\hline & & & & $\mathrm{Z} 12070$ & T. sp. 'black' & Ht_55 & \\
\hline Kisokwe & $-4,24$ & 29,18 & 890 & KX513593 & T. sp. 'black' & Ht_53* & 890 \\
\hline Lubumba & $-3,52$ & 29,15 & 865 & KX513594 & T. sp. 'black' & Ht_52 & 865 \\
\hline \multirow[t]{2}{*}{ Luhanga } & $-3,5186$ & 29,3969 & 14403 & KX513721 & T. sp. 'black' & Ht_01 & KS24B8 \\
\hline & & & 14456 & KX513669 & T. sp. 'black' & Ht_01 & T14/03/A6 \\
\hline
\end{tabular}


Table 1 continued

\begin{tabular}{|c|c|c|c|c|c|c|c|}
\hline Location & Latitude (S) & Longitude (E) & Extraction no. & Accession no. & Species & Haplotype & Sample ID \\
\hline & & & 14405 & KX513719 & T. sp. 'black' & Ht_09 & KS24B8 \\
\hline & & & 14406 & KX513718 & T. sp. 'black' & Ht_10 & KS24B8 \\
\hline & & & 14407 & KX513717 & T. sp. 'black' & Ht_11 & KS24B8 \\
\hline & & & 14457 & KX513668 & T. sp. 'black' & Ht_11 & T14/03/A7 \\
\hline & & & 14458 & KX513667 & T. sp. 'black' & Ht_27 & T14/03/A8 \\
\hline & & & 14459 & KX513666 & T. sp. 'black' & Ht_28 & T14/03/A9 \\
\hline & & & 14460 & KX513665 & T. sp. 'black' & Ht_29 & $\mathrm{T} 14 / 03 / \mathrm{A} 10$ \\
\hline & & & 14462 & KX513663 & T. sp. 'black' & Ht_29 & $\mathrm{T} 14 / 03 / \mathrm{B} 2$ \\
\hline & & & 14461 & KX513664 & T. sp. 'black' & Ht_30 & T14/03/B1 \\
\hline & & & 14467 & KX513658 & T. sp. 'black' & Ht_30 & $\mathrm{T} 14 / 03 / \mathrm{B} 7$ \\
\hline & & & 14463 & KX513662 & T. sp. 'black' & Ht_31 & T14/03/B3 \\
\hline & & & 14464 & KX513661 & T. sp. 'black' & Ht_32 & $\mathrm{T} 14 / 03 / \mathrm{B} 4$ \\
\hline & & & 14465 & KX513660 & T. sp. 'black' & Ht_33 & $\mathrm{T} 14 / 03 / \mathrm{B} 5$ \\
\hline & & & 14466 & KX513659 & T. sp. 'black' & Ht_34 & $\mathrm{T} 14 / 03 / \mathrm{B} 6$ \\
\hline & & & 14468 & KX513657 & T. sp. 'black' & Ht_35 & T14/03/B8 \\
\hline & & & 14470 & KX513605 & T. sp. 'black' & Ht_35 & $\mathrm{T} 14 / 03 / \mathrm{B} 10$ \\
\hline & & & 14469 & KX513656 & T. sp. 'black' & Ht_36 & T14/03/B9 \\
\hline & & & 14471 & KX513655 & T. sp. 'black' & Ht_37 & $\mathrm{T} 14 / 03 / \mathrm{C} 1$ \\
\hline & & & 14404 & KX513720 & T. sp. 'black' & Ht_01 & KS24B8 \\
\hline & & & 14408 & KX513716 & T. sp. 'black' & Ht_11 & KS24D6 \\
\hline & & & 14409 & KX513715 & T. sp. 'black' & Ht_11 & KS24D6 \\
\hline & & & 14410 & KX513714 & T. sp. 'black' & Ht_11 & KS24D6 \\
\hline & & & 783 & KX513602 & T. sp. 'black' & Ht_27 & 783 \\
\hline & & & 784 & KX513601 & T. sp. 'black' & Ht_27 & 784 \\
\hline & & & 787 & KX513598 & T. sp. 'black' & Ht_09 & 787 \\
\hline & & & 785 & KX513600 & T. sp. 'black' & Ht_11* & 785 \\
\hline & & & 786 & KX513599 & T. sp. 'black' & Ht_27* & 786 \\
\hline \multirow[t]{17}{*}{ Magara } & $-3,726$ & 29,31 & 14395 & KX513729 & T. sp. 'black' & Ht_05 & KS20G7 \\
\hline & & & 14486 & KX513640 & T. sp. 'black' & Ht_05 & T14/03/D6 \\
\hline & & & 14396 & KX513728 & T. sp. 'black' & Ht_06 & KS20G7 \\
\hline & & & 14401 & KX513723 & T. sp. 'black' & Ht_06 & KS20G8 \\
\hline & & & 14483 & KX513643 & T. sp. 'black' & Ht_06 & T14/03/D3 \\
\hline & & & 14485 & KX513641 & T. sp. 'black' & Ht_06 & T14/03/D5 \\
\hline & & & 14490 & KX513636 & T. sp. 'black' & Ht_06 & T14/03/D10 \\
\hline & & & 14397 & KX513727 & T. sp. 'black' & Ht_07 & KS20G7 \\
\hline & & & 14399 & KX513725 & T. sp. 'black' & Ht_07 & KS20G7 \\
\hline & & & 14484 & KX513642 & T. sp. 'black' & Ht_07 & T14/03/D4 \\
\hline & & & 14488 & KX513638 & T. sp. 'black' & Ht_07 & T14/03/D8 \\
\hline & & & 14491 & KX513635 & T. sp. 'black' & Ht_07 & $\mathrm{T} 14 / 03 / \mathrm{E} 1$ \\
\hline & & & 14398 & KX513726 & T. sp. 'black' & Ht_08 & KS20G7 \\
\hline & & & 14402 & KX513722 & T. sp. 'black' & Ht_08 & KS20G8 \\
\hline & & & 14487 & KX513639 & T. sp. 'black' & Ht_39 & T14/03/D7 \\
\hline & & & 14489 & KX513637 & T. sp. 'black' & Ht_39 & T14/03/D9 \\
\hline & & & 14400 & KX513724 & T. sp. 'black' & Ht_07 & KS20G8 \\
\hline
\end{tabular}


Table 1 continued

\begin{tabular}{|c|c|c|c|c|c|c|c|}
\hline Location & Latitude (S) & Longitude (E) & Extraction no. & Accession no. & Species & Haplotype & Sample ID \\
\hline & & & 14482 & KX513644 & T. sp. 'black' & Ht_07* & $\mathrm{T} 14 / 03 / \mathrm{D} 2$ \\
\hline Mboko & $-3,9166$ & 29,083 & & AY660763 & T. sp. 'black' & Ht_50 & \\
\hline Minago & -4 & 29,4166 & & $\mathrm{Z75700}$ & T. sp. 'black' & Ht_16 & \\
\hline \multirow[t]{24}{*}{ Muguruka } & $-4,244$ & 29,3716 & 14509 & KX513618 & T. brichardi & Ht_12 & T14/03/F8 \\
\hline & & & 14517 & KX513611 & T. brichardi & Ht_12 & T14/03/G6 \\
\hline & & & 14510 & KX513617 & T. brichardi & Ht_12 & T14/03/F9 \\
\hline & & & 14511 & KX513616 & T. brichardi & Ht_12 & $\mathrm{T} 14 / 03 / \mathrm{F} 10$ \\
\hline & & & 14512 & KX513615 & T. brichardi & Ht_12 & T14/03/G1 \\
\hline & & & 14513 & KX513614 & T. brichardi & Ht_12 & $\mathrm{T} 14 / 03 / \mathrm{G} 2$ \\
\hline & & & 14514 & KX513613 & T. brichardi & Ht_12 & T14/03/G3 \\
\hline & & & 14443 & KX513682 & T. brichardi & Ht_12 & KS25E2 \\
\hline & & & 14445 & KX513680 & T. brichardi & Ht_12 & KS25E2 \\
\hline & & & 14449 & KX513676 & T. brichardi & Ht_12 & KS25G6 \\
\hline & & & 14450 & KX513675 & T. brichardi & Ht_13 & KS25G6 \\
\hline & & & 14505 & KX513621 & T. brichardi & Ht_22 & T14/03/F5 \\
\hline & & & 14520 & KX513608 & T. brichardi & Ht_22 & T14/03/G9 \\
\hline & & & 14444 & KX513681 & T. brichardi & Ht_22 & KS25E2 \\
\hline & & & 14447 & KX513678 & T. brichardi & Ht_23 & KS25E3 \\
\hline & & & 14518 & KX513610 & T. brichardi & $\mathrm{Ht} \_24$ & $\mathrm{~T} 14 / 03 / \mathrm{G} 7$ \\
\hline & & & 14448 & KX513677 & T. brichardi & $\mathrm{Ht} \_24$ & KS25G6 \\
\hline & & & 14504 & KX513622 & T. brichardi & Ht_41 & $\mathrm{T} 14 / 03 / \mathrm{F} 4$ \\
\hline & & & 14519 & KX513609 & T. brichardi & Ht_41 & T14/03/G8 \\
\hline & & & 14507 & KX513619 & T. brichardi & Ht_42 & $\mathrm{T} 14 / 03 / \mathrm{F} 7$ \\
\hline & & & 14515 & KX513612 & T. brichardi & Ht_43 & $\mathrm{T} 14 / 03 / \mathrm{G} 4$ \\
\hline & & & 14521 & KX513607 & T. brichardi & Ht_44 & $\mathrm{T} 14 / 03 / \mathrm{G} 10$ \\
\hline & & & 14506 & KX513620 & T. brichardi & Ht_22 & T14/03/F6 \\
\hline & & & 14446 & KX513679 & T. brichardi & Ht_12 & $\mathrm{KS} 25 \mathrm{E} 2$ \\
\hline \multirow[t]{2}{*}{ Mvugo } & $-3,9808$ & 29,503 & 14411 & KX513713 & T. brichardi & Ht_12 & $\mathrm{KS} 25 \mathrm{H} 7$ \\
\hline & & & 14412 & KX513712 & T. brichardi & Ht_13 & $\mathrm{KS} 25 \mathrm{H} 7$ \\
\hline \multirow[t]{3}{*}{ Ngombe } & $-4,666$ & 29,6166 & & AJ489622 & T. brichardi & Ht_01 & \\
\hline & & & & AJ295923 & T. brichardi & Ht_57 & \\
\hline & & & & AJ95924 & T. brichardi & Ht_58 & \\
\hline \multirow[t]{4}{*}{ Nyahurongoka } & $-3,69$ & 29,33 & 14414 & KX513710 & T. sp. 'black' & Ht_06 & $\mathrm{KS} 20 \mathrm{~A} 2$ \\
\hline & & & 14413 & KX513711 & T. sp. 'black' & Ht_14 & KS20I2 \\
\hline & & & 1386 & KX513603 & T. sp. 'black' & Ht_07 & 1386 \\
\hline & & & 1387 & KX513592 & T. sp. 'black' & Ht_54 & 1387 \\
\hline \multirow[t]{4}{*}{ Nyanza Lac } & $-4,33$ & 29,583 & 14969 & KX513742 & T. brichardi & Ht_46 & KS32B1 \\
\hline & & & & Z12054 & T. brichardi & Ht_46 & \\
\hline & & & 14968 & KX513741 & T. brichardi & Ht_46* & KS32A9 \\
\hline & & & 14967 & KX513740 & T. brichardi & Ht_46* & KS32A8 \\
\hline Rutunga & $-3,666$ & 29,316 & 14522 & KX513606 & T. sp. 'black' & Ht_45 & $\mathrm{T} 14 / 03 / \mathrm{H} 1$ \\
\hline
\end{tabular}


Table 1 continued

\begin{tabular}{|c|c|c|c|c|c|c|c|}
\hline Location & Latitude (S) & Longitude (E) & Extraction no. & Accession no. & Species & Haplotype & Sample ID \\
\hline & & & & $\mathrm{Z} 12050$ & T. sp. 'black' & Ht_49 & \\
\hline & & & & Z12051 & T. sp. 'black' & Ht_06 & \\
\hline & & & & Z12049 & T. sp. 'black' & Ht_49 & \\
\hline \multirow[t]{4}{*}{ 'Ubwari-green' } & $-4,164$ & 29,2582 & & AY660840 & T. cf. brichardi & Ht_56 & \\
\hline & & & & AY660842 & T. sp. 'black' & Ht_56 & \\
\hline & & & & AY660843 & T. sp. 'black' & Ht_56 & \\
\hline & & & 13859 & KX513591 & T. sp. 'black' & Ht_56* & T13/1/B1 \\
\hline $\begin{array}{l}\text { Ubwari Penninsula } \\
\text { (color morph } \\
\text { not known) }\end{array}$ & & & & AY660841 & $T . \mathrm{sp}$. & Ht_48 & \\
\hline
\end{tabular}

Individuals marked in bold letters and asterisks were also used in the AFLP tree

of a larger dataset (Van Steenberge, unpublished data) in which negative controls and a minimum of 19 replicates per primer combination were included in the reactions. Size and peak height of fragments between 100 and 500 bp were determined using GeneMapper v.3.7 (Applied Biosystems). Bins were checked by eye and preprocessed for threshold optimization for locus retention and phenotype calling with AFLP-SCORE 1.4a (Whitlock et al., 2008) following (Mattersdorfer et al., 2012). The average mismatch error rate was $1.26 \%$. The final binary matrix for the representative set of northern Tropheus consisted of 442 polymorphic characters.

\section{Phylogenetic analysis}

Phylogenetic relationships among mitochondrial haplotypes were inferred by means of a neighborjoining (NJ) tree in MEGA 6.0 (Tamura et al., 2013). For NJ tree inference, identical sequences were collapsed into haplotypes using DnaSP 5.10 (Rozas, 2010). Based on the Bayesian information criterion (BIC), jModelTest 0.1 (Posada, 2008) identified the HKY+G (Hasegawa et al., 1985a, b) model as the best fitting model of molecular evolution. As this model is not implemented in MEGA, the TN93+G (Tamura \& Nei, 1993) model-as the best fitting model available in MEGA — was employed for NJ tree inference instead. Nodal support was assessed by means of bootstrapping (10,000 pseudoreplicates). Furthermore, a statistical parsimony network (Templeton et al., 1992) was constructed in POPART (Leigh and Bryant, 2015).

To get an idea about the putative timing of major divergence events among and within the main mitochondrial lineages, we inferred a time-calibrated mitochondrial tree in BEAST 1.8 (Drummond \& Rambaut, 2007). Two independent MCMC chains were run for $10^{6}$ generations, with model parameters and trees sampled every 1000 generations. We employed the HKY $+\mathrm{G}$ substitution model with a strict molecular clock (as we are looking at mostly intraspecific data; Brown \& Yang, 2011) assuming minimum and maximum substitution rates of 3.24 and $5.7 \%$ per MY, respectively (Koblmüller et al., 2009; Genner et al., 2010), and a Bayesian skyline tree prior (Drummond et al., 2005). All other priors were left at default. The first $10 \%$ of generations were discarded from each log and tree file as burn-in before the two chains were combined using LogCombiner (available as part of the BEAST package). Chain convergence to stationarity for all model parameters was assessed in Tracer 1.6 (available from http://beast.bio.ed.ac.uk/tracer). The pooled post-burn-in Effective Sample Sizes (ESS) for all parameters exceeded 200, indicating that the pooled $\log$ file accurately represented the posterior distribution (Kuhner, 2009). Divergence times were derived from the pooled post-burn-in results and TreeAnnotator (available as part of the BEAST package) was used to compute a maximum-clade-credibility tree, which was visualized in FigTree 1.4.1 (available from http://beast. bio.ed.ac.uk/figtree). Divergence times were calculated as median node heights of the $95 \%$ highest posterior density (HPD) intervals.

HExT (Schneider et al., 2016) was used to infer a $\mathrm{NJ}$ tree from the AFLP data based on Nei-Li distances (Nei \& Li, 1979) and estimate statistical support from 1000 bootstrap replicates. The tree was rooted with 
Tropheus duboisi, which was previously shown to represent the sister group of all other Tropheus (Koblmüller et al., 2010).

Within- and among-population patterns of genetic diversity

Genetic diversity indices-number of haplotypes $(H)$, Haplotype diversity $\left(H_{d}\right)$, nucleotide diversity $(\pi)$-as well as mismatch distributions were calculated in DnaSP for all populations with a sample size $N \geq 14$. Population differentiation was estimated by $\theta_{S T}$ (Weir \& Cockerham, 1984) and $\Phi_{S T}$ (Excoffier et al., 1992) in Arlequin v.3.5 (Excoffier \& Lischer, 2010), with significance inference corrected for multiple testing following (Benjamini \& Hochberg, 1995).

Past population size trajectories for main mitochondrial lineages and populations (with sample size $N \geq 9$ ) were inferred by means of Bayesian skyline plots (BSPs; Drummond et al., 2005) in BEAST and visualized in Tracer, employing the same settings as for the time-calibrated mitochondrial tree (see above). The various datasets required different run lengths, but all analyses were run until ESS for all parameters were $>200$.

\section{Results}

Phylogeographic patterns

The combined analysis of 179 individuals from 21 localities in Congo and Burundi identified 60 haplotypes and corroborates the assignment of all Tropheus from this area to two major mtDNA lineages (Fig. 2), TCS-lineages 1-A and 2-B (following Sturmbauer et al., 2005). Moreover, each of the mitochondrial clusters comprises two to three subclusters, coded in

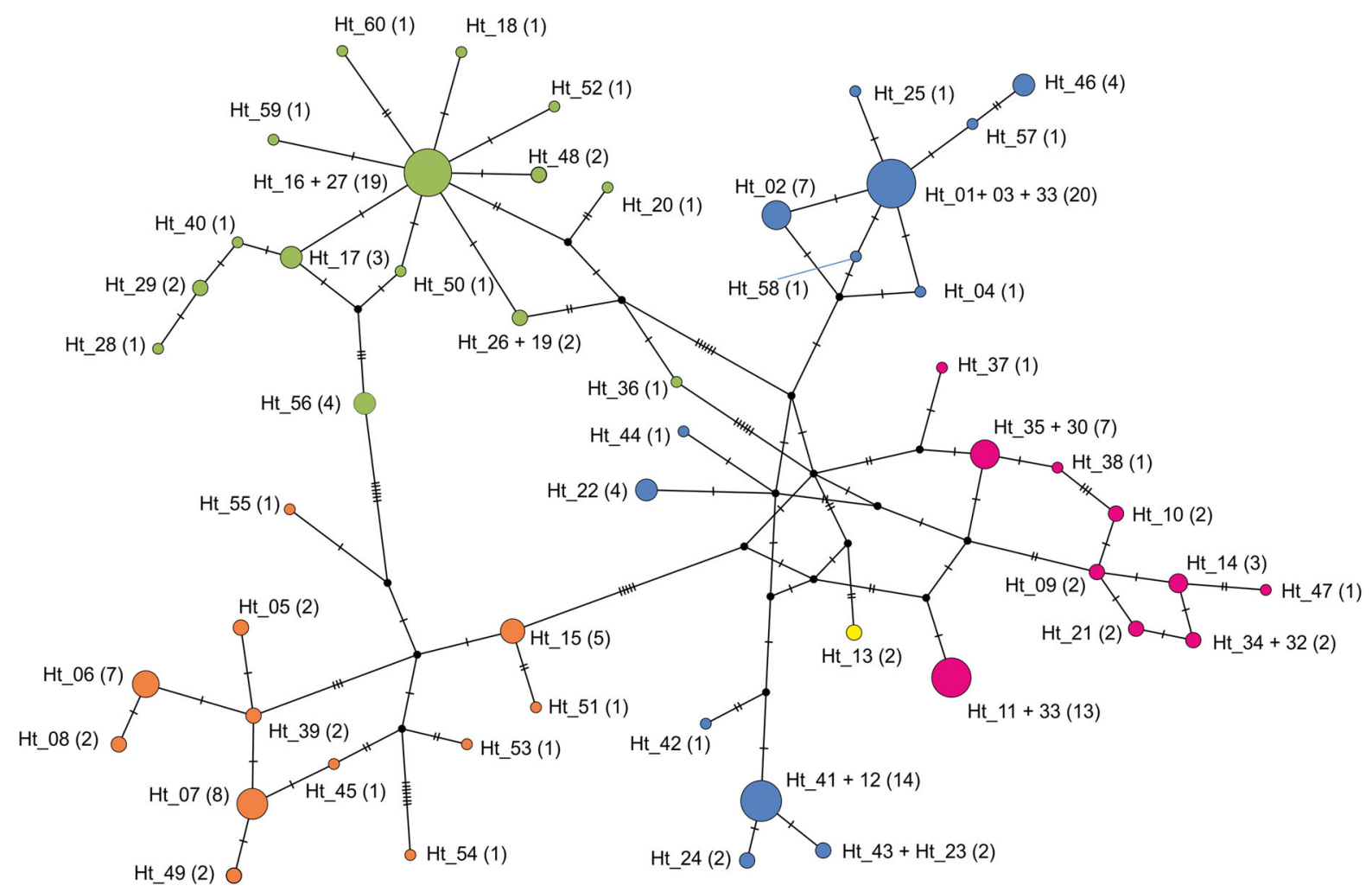

Fig. 2 Statistical parsimony network of northern Tropheus haplotypes based on a 352 bp long segment of the mitochondrial control region. Each haplotype is represented by a circle, the size of which correlates with the number of individuals sharing the same haplotype. Small bars indicate the number of substitutions between haplotypes. Each of the mitochondrial clusters comprises two to three subclusters, coded in yellow, blue and pink for TCS-lineage 1, and in green and red for TCSlineage 2 in all figures 


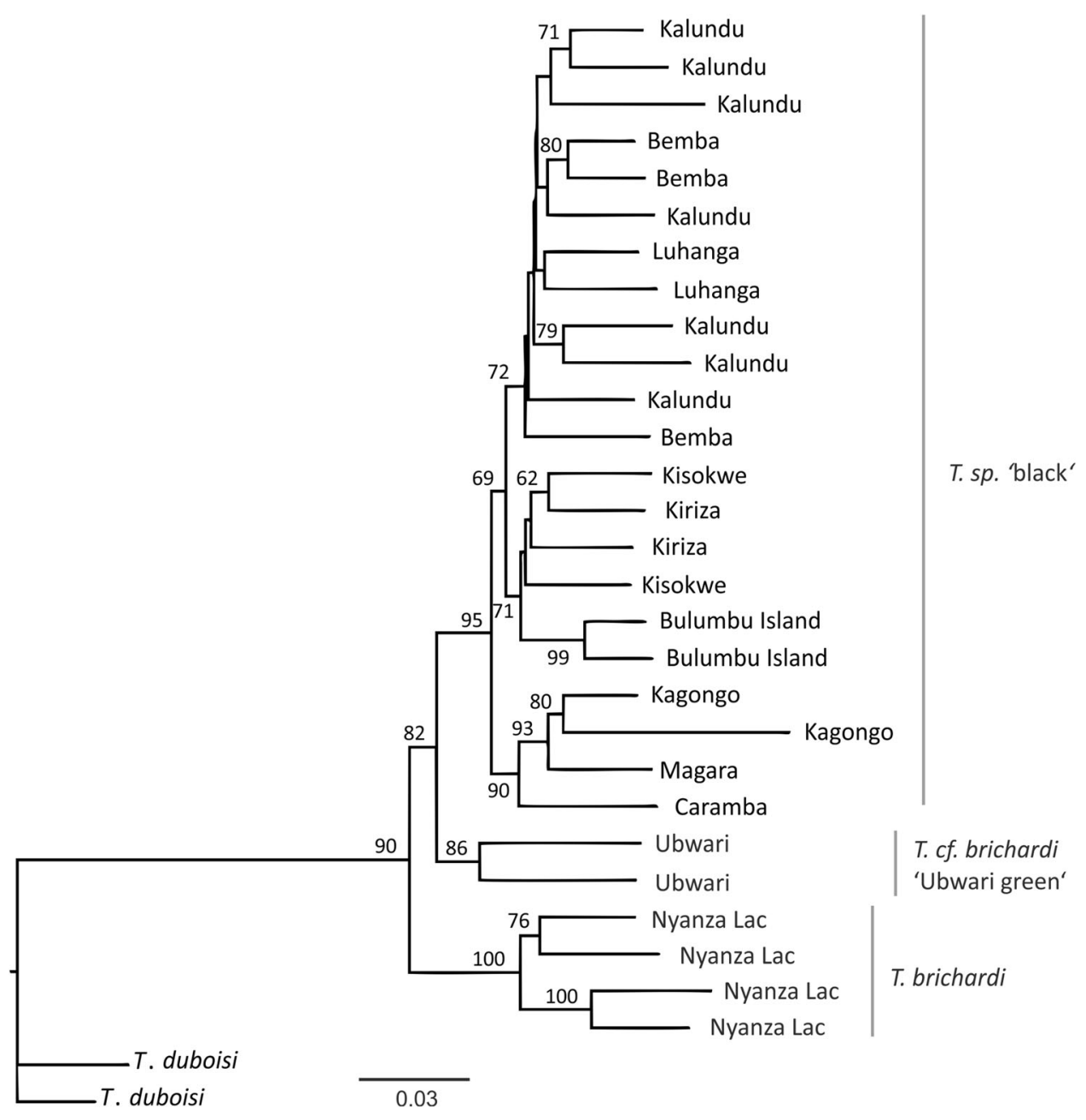

Fig. 3 NJ tree based on 442 polymorphic AFLP loci. Only bootstrap values $>50$ are shown. Tropheus duboisi was used as outgroup

yellow, blue and pink for TCS-lineage 1, and in green and red for TCS-lineage 2 in all Figures. While the mtDNA tree is partially inconsistent with the current species assignment, the AFLP-based nuclear tree shows the reciprocal monophyly of the two Tropheus species present in the northernmost part of the Lake, $T$. brichardi and $T$. sp. 'black,' in relation to the outgroup T. duboisi (Fig. 3). While the individuals of $T$. sp. 'black' Caramba are resolved in the clade of all other $T$. sp. 'black,' the phenotypically aberrant Tropheus cf. brichardi 'Ubwari-green' from Cape Muzimu southwards occupy an intermediate position in the AFLPtree (Fig. 3, note that one previously published sample from the Ubwari Penninsula was not explicitly assigned to either of the two taxonomic entities; it is thus labeled as $T$. sp. Ubwari Penninsula in Table 1 and the figures). The time-calibrated mitochondrial tree (Fig. 4) suggests that TCS-lineages 1 and 2 diverged roughly 450-800 KYA and indicates simultaneous east/west divergence in three out of the five mitochondrial sublineages about 150-265 KYA. The presence of shared or closely related haplotypes on opposite shores (in the yellow sublineage the sample size was too small) indicated gene flow between eastern and western populations in the more recent past. Several populations comprised haplotypes pertaining to different haplogroups (see haplogroup distributions along shoreline in Fig. 5, and mismatch distributions in Supplementary Fig. 1). Concerning the distribution of haplotype lineages, one can clearly observe that along the eastern shoreline northwards the distribution of populations with blue and pink 


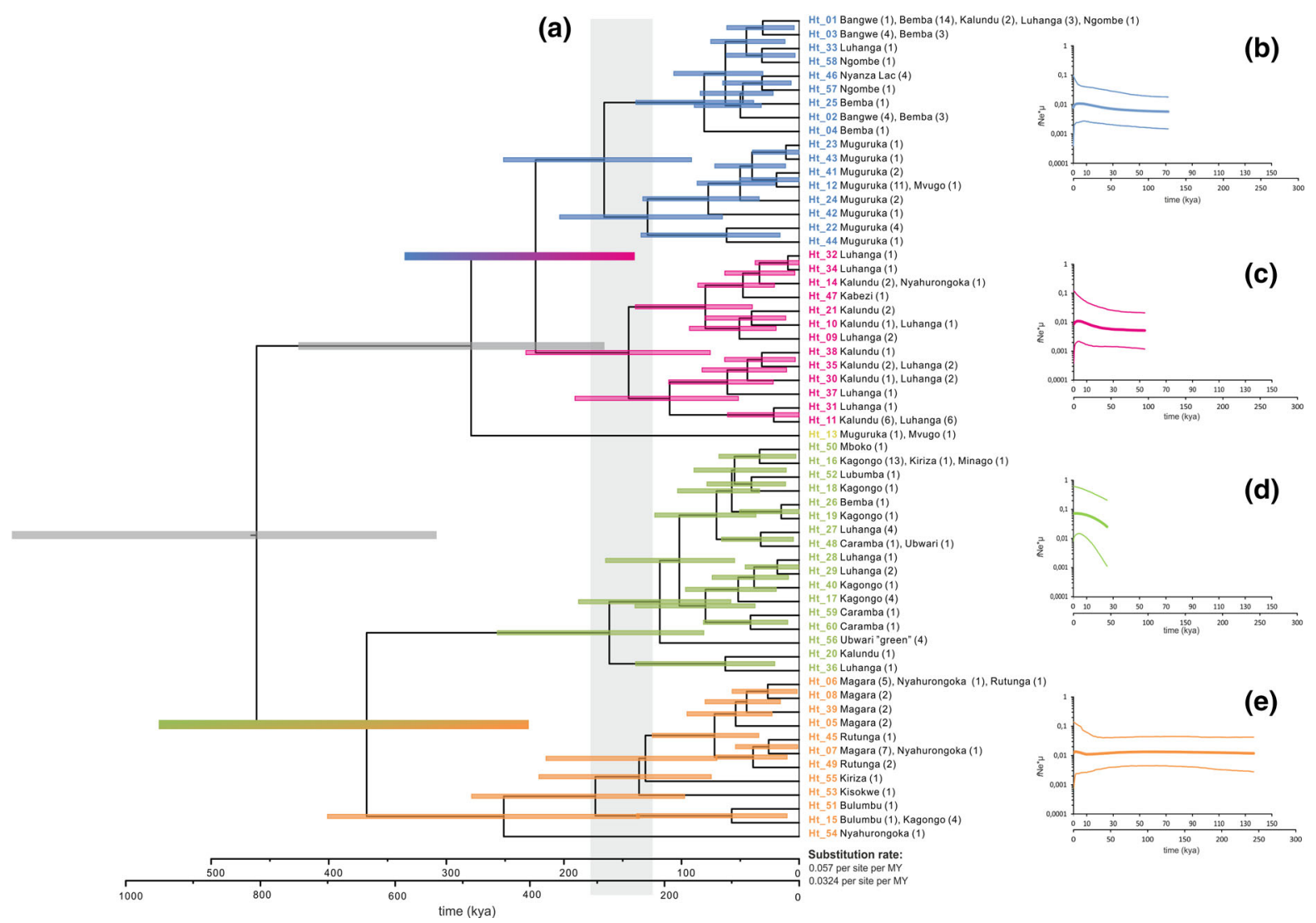

Fig. 4 Chronogram of the diversification of northern Tropheus based on mitochondrial control region haplotypes. Divergence time estimates are represented as the median node height of the 95\% highest posterior density (HPD) interval from a BEAST maximum-clade-credibility tree. Node bars span the 95\% HPD interval for each node. The colors indicate major haplotype lineages comprising two to three subclusters, coded in yellow,

haplotypes (TCS-1) is interrupted by populations with green and orange haplotypes (TCS-2), and that blue and pink haplotypes (TCS-1) again dominate the very north of the lake. The green and orange TCS-2 haplotypes, however, dominate the western shores at the Ubwari Penninsula, to be progressively replaced northwards by blue TCS-1 haplotypes at Bemba/ Bangwe and blue/pink haplotypes at Luhanga/ Kalundu. From the distribution of identical and very closely related haplotypes (1-2 mutations), one can deduce recent long-distance dispersal of blue haplotypes from Nyanza Lac to the Bemba-Kalundu stretch, as well as intense connections of green and orange haplotypes across the ridge at the eastern and western Ubwari Faults (Fig. 5). The two sympatric Tropheus, $T$. sp. 'black' Caramba and T. cf. brichardi 'Ubwari- blue, and pink for TCS-lineage 1, and in green and red for TCSlineage 2 (Fig. 2). Insets to the right $\mathbf{b}-\mathbf{e}$ show past population size changes for the four major mitochondrial clades, as inferred by means of Bayesian skyline plots. The $y$-axis represents the population size parameter (female effective population size times the mutation rate). Thick and thin lines denote median estimates and 95\% HPD intervals, respectively

green', share the same mtDNA lineage (TCS-2), despite their different positions in the AFLP tree (Fig. 3), suggesting hybridization and subsequent mitochondrial capture. The BSPs revealed signatures of Late Pleistocene population growth in all but the orange subcluster (Fig. 4). Particularly strong population expansion is evident for the green subcluster. Whether the recent population size decline (over last few hundreds of years) apparent in most mitochondrial lineages is a true signal potentially correlated with increasing human population sizes along the lake shore or simply a methodological artifact (Chikhi et al., 2010; Heller et al., 2013) remains unclear.

It is also interesting to note that the TCS-1 haplotypes assigned to the pink haplotype cluster are not exclusive to the very northern part of the lake. This 


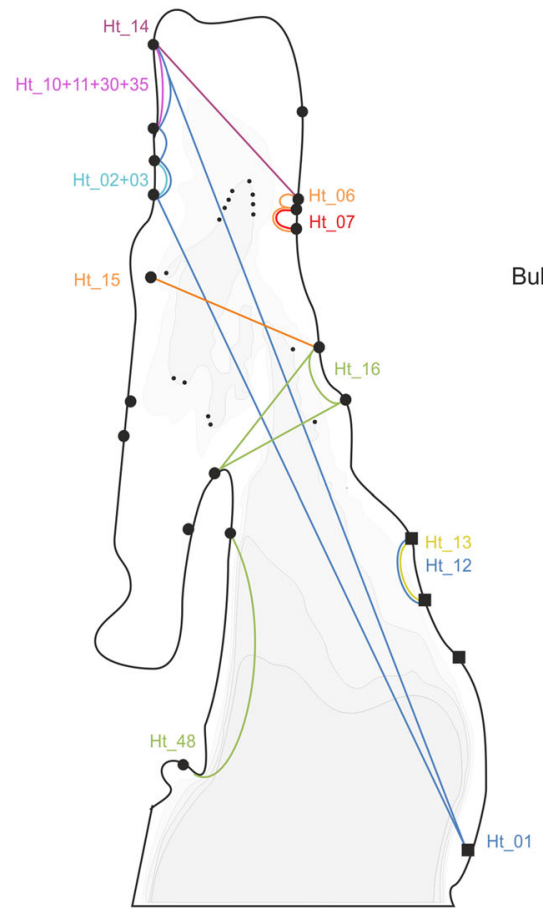

(a) identical haplotypes

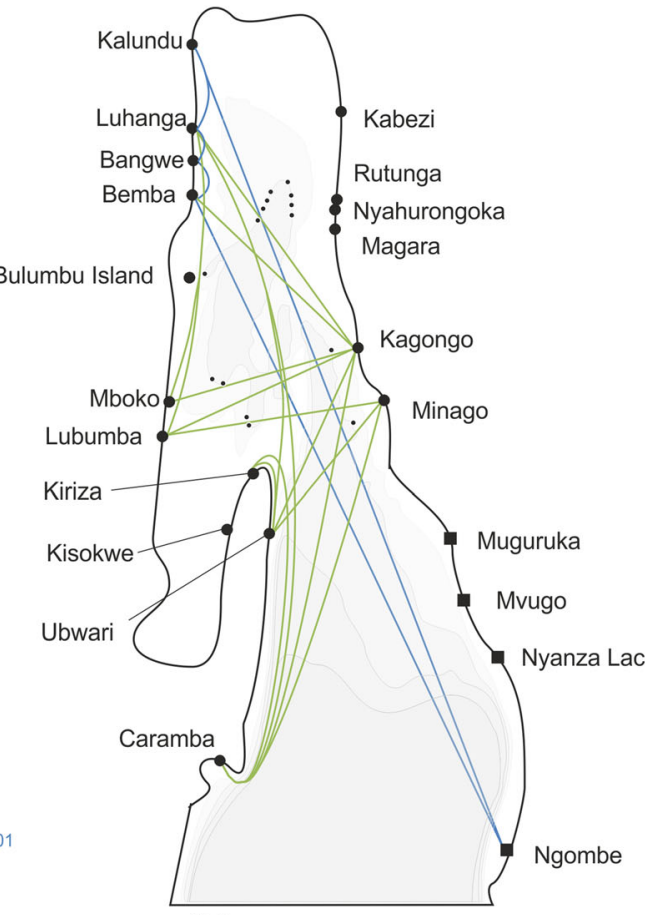

(b) plus 1 mutation

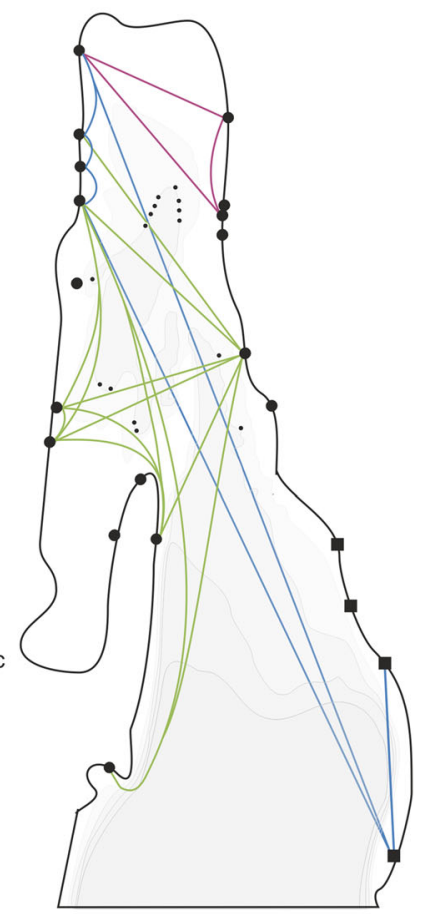

(c) plus 2 mutations
Fig. 5 Distribution of identical and similar (1-2 mutations difference) Tropheus haplotypes in northern Lake Tanganyika. Colors are assigned to the clades as described in the text.

haplogroup is also found in some individuals of $T$. moorii from the southern basin such at Fulwe and Wapembe, as well as in fish at Kasakalawe from the very southern end of the lake. In-between, we only have a record of the pink haplotype cluster in a single $T$. sp. 'black' Kirschfleck individual from Mabilibili at the central eastern shore. These biogeographic data suggest a particularly widespread migration of the members of this TCS-sublineage (Sturmbauer et al., 2005).

\section{Population genetics}

The number of haplotypes per population varied considerably among populations, with the lowest number detected in Magara $(N=5)$ and the highest number detected in Luhanga $(N=11)$. Estimates of haplotype diversity $\left(H_{d}\right)$ and nucleotide diversity $(\pi)$ ranged from 0.674 (Kagongo) to 0.897 (Luhanga) and 0.00406 (Magara) to 0.02420 (Luhanga) (Table 2). The large variation of $\pi$ across populations indicates that different numbers of haplogroups contributed to the genetic diversity in the different populations.
Squares marking sampling sites indicate the occurrence of $T$. brichardi instead of $T$. sp. 'black' (circle symbols)

Population genetic differentiation was high and highly significant for most pairwise comparisons. Only the two northernmost populations at the western shoreline were not significantly differentiated from each other (Table 2). The BSPs revealed clear signatures of simultaneous recent population growth for four populations (Bemba, Bangwe, Magara, Kagongo), whereas the three other populations (Luhanga, Kalundu, Muguruka) appear to have experienced a decline in the recent past (Fig. 6).

\section{Discussion}

\section{Phylogeography}

Our new data fill important gaps and allow us to hypothesize a colonization and admixis scenario for Tropheus populations inhabiting the very northern section of Lake Tanganyika, and highlight the combined effect of recurrent drastic lake level fluctuations and a complex basin structure on the phylogeographic structure of rock-dwelling cichlids in this part of the 
Table 2 Population sample sizes $(N)$, genetic diversity and pairwise population genetic differentiation based on the most variable part of the mitochondrial control region

\begin{tabular}{|c|c|c|c|c|c|c|c|c|c|c|}
\hline & $N$ & $H$ & $H_{\mathrm{d}}$ & $\pi$ & Bemba & Luhanga & Kalundu & Magara & Kagongo & Muguruka \\
\hline Bemba & 14 & 6 & 0.736 & 0.00652 & & $0.113 * * *$ & $0.144 * *$ & $0.242 * * *$ & $0.298 * * *$ & $0.244 * * *$ \\
\hline Luhanga & 29 & 11 & 0.897 & 0.02420 & $0.311 * * *$ & & 0.002 & $0.142 * * *$ & $0.194 * * *$ & $0.147 * * *$ \\
\hline Kalundu & 18 & 8 & 0.863 & 0.01634 & $0.489 * * *$ & 0.037 & & $0.173 * * *$ & $0.230 * * *$ & $0.178 * * *$ \\
\hline Magara & 18 & 5 & 0.778 & 0.00406 & $0.882 * * *$ & $0.625 * * *$ & $0.755 * * *$ & & $0.277 * * *$ & $0.225 * * *$ \\
\hline Kagongo & 24 & 6 & 0.674 & 0.01114 & $0.683 * * *$ & $0.368 * * *$ & $0.605 * * *$ & $0.785 * * *$ & & $0.277 * * *$ \\
\hline Muguruka & 24 & 7 & 0.688 & 0.01151 & $0.676^{* * *}$ & $0.419 * * *$ & $0.504 * * *$ & $0.844 * * *$ & $0.757 * * *$ & \\
\hline
\end{tabular}

Only populations with $N \geq 14$ are included

$H$ number of haplotypes, $H_{d}$ gene diversity, $\pi$ nucleotide diversity. Above diagonal: $\theta_{\mathrm{ST}}$ estimates, below diagonal: $\Phi_{\mathrm{ST}}$ values. Significance levels, $P<0.05,<0.01$ and $<0.001$, after correction for multiple test, are indicated as *, **, and ***, respectively

Fig. 6 Bayesian skyline plots of population sizes through time for populations with a sample size $N \geq 9$. Depicted are the median estimates. The $y$-axis represents the population size parameter (female effective population size times the mutation rate)

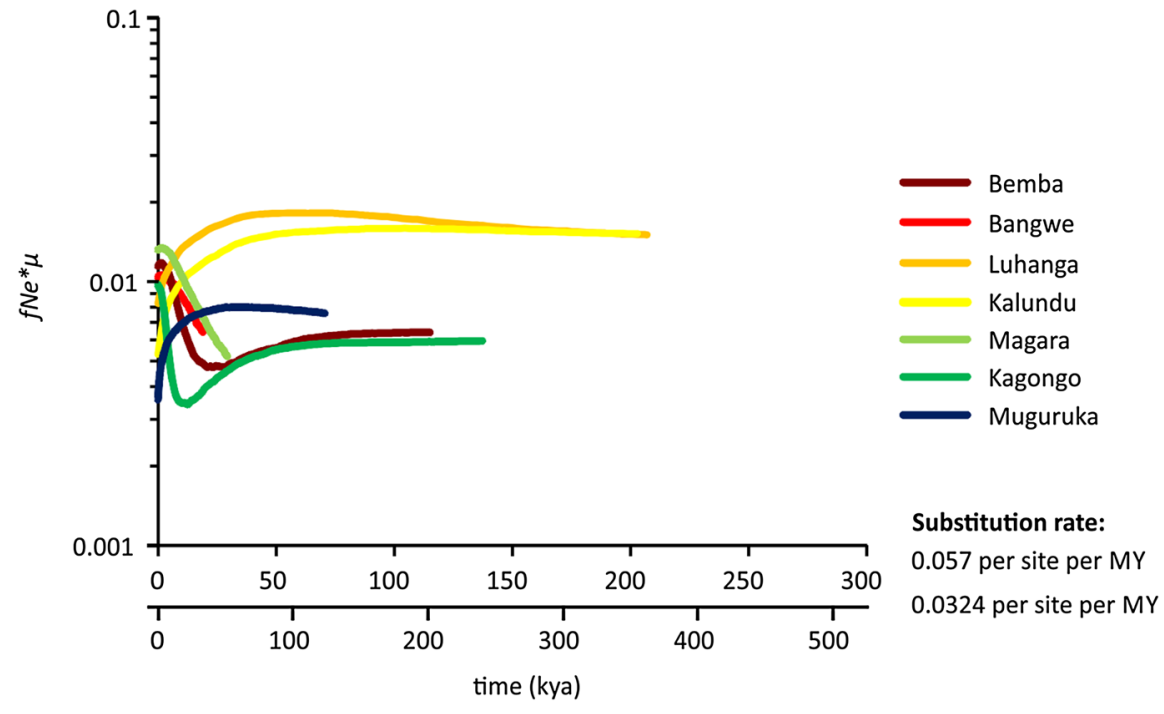

lake (Cohen et al., 2007). This region comprises a small subbasin at minus $300 \mathrm{~m}$ north of the Ubwari Penninsula, named the Bujumbura basin, separated by a ridge from the Kigoma basin. This basin played an important role as lowstand refugium and melting pot for two mtDNA lineages of Tropheus in the more recent past. Our data suggest that it seems most likely that the original Tropheus population of the Bujumbura basin comprised $T$. sp. 'black,' given that all populations inhabiting this basin are assigned to this species. The original $T$. sp. 'black' population was substantially perturbated by a series of lake level fluctuations, triggering the invasion of $T$. brichardi from the Kigoma basin along the eastern shoreline. At the eastern shore of the Bujumbura basin the presentday distribution of identical or closely related mtDNA haplotypes assigned to the T. brichardi lineage (TCS1) is discontinuous. As the pink TCS-1 haplotype sublineage is exclusively distributed in the very north of the Bujumbura basin and shows great diversity only there, while the blue TCS-1 haplotype sublineage is equally diverse but much more widespread, ranging from the very northwest of the present-day lake down to Muguruka and even Ngombe, we suggest that it is more parsimonious to assume (at least) two migration waves for the $T$. brichardi-lineage. The members of the pink TCS-1 haplotypes are likely to have arrived at the first wave. In-between the two migration waves, during another period of lower lake level, T. sp. 'black' with TCS-2 haplotypes crossed over from the west along the Ubwari ridge and largely replaced the T. brichardi- like TCS-1 populations from Minago to 
Magara. That their colonization of the eastern shores between Minago and Magara is not very recent can be delineated from the evolution of two distinct TCS-2 haplotype subgroups, one with its center of diversity at the eastern and the other at the western shore. At least one subsequent re-connection blurred the distribution pattern of TCS-2 haplotypes and allowed large-scale admixis with TCS-1 haplotypes from the blue sublineage. That this admixis event was very recently, probably during the last glacial maximum, can be seen from the long-distance distribution of identical and very closely related haplotypes in both lineages (Fig. 5). This (re)colonization by $T$. brichardi might have been successful due to a local deterioration of the habitat. Both the volcanic activity in the Bemba area (Pflumio et al., 1994) and the erratic flow of the sediment carrying Rusizi River (Cassanova \& Hillaire-Marcel, 1992) could be responsible for this.

Albeit all populations of the Bujumbura basin are $T$. sp. 'black,' despite of the complex phylogeographic pattern of TCS-1 and TCS-2 haplotypes, many seem heavily introgressed by $T$. brichardi TCS- 1 haplotypes and sometimes phenotypically intermediate (Van Steenberge, 2014). This is also supported by the presence of TCS-1 haplotypes at Nyahurongoka and Kabezi. Since the rock shores around the Ubwari Peninsula are dominated by TCS-2 haplotypes, despite the partial sympatry of two species, we suggest that this section represents their original distribution area, and thus the original distribution area of $T$. sp. 'black.' It seems likely that the TCS-2 haplotypes sampled from Minago to Rutunga, as they interrupt the distribution of TCS-1 haplotypes there, made it across the bottleneck area northeast of Cape Banza between the West and East Ubwari Faults. The lowstandshoreline in this area over the ridge is extremely narrow and resembles a meandric river, so that complex admixis scenarios along both basin edges are inevitable. This may have allowed repeated bridging of mainly TCS-2 haplotypes (see Fig. 1 in Lezzar et al., 2002). A complex colonization-admixispartial extinction scenario is also supported by the present-day distribution of the more distantly related Tropheus duboisi, which occurs almost continuously together with TCS-1 haplotypes of $T$. brichardiprovenience at the east coast from the Mahale Mountains to Muguruka, but only occurs at a single small stretch at the NW coast near Bemba, together with a TCS-1 dominated Tropheus sp. 'black' population sharing identical haplotypes with their allies at Ngombe. In fact, the control region haplotypes in the T. duboisi populations from Bemba and Mwamugongo (near Ngombe) are separated by 2 mutations only (Van Steenberge et al., 2015). Moreover, Julidochromis species show similar cross-bottleneck distribution, in that $J$. regani shares the habitat with "pure" T. brichardi around Nyanza Lac, followed northwards by a putative hybrid between $J$. regani and J. marlieri (J. regani affinis from Rumonge northwards, as well as at the tip of the Ubwari Penninsula), followed by pure J. marlieri around Rutunga, and finally followed by pure $J$. regani at Bujumbura again (Brichard, 1978).

Concerning the lake-wide distribution patterns of the TCS-1 haplotype lineage and its subclusters, it is important to note that the pink TCS- 1 subcluster is very widespread but rare (Sturmbauer et al., 2005). Aside of the very North of the lake it only occurs in a single individual at Mabilibili, in populations at Fulwe and north of Wapembe, and at Kasakalawe at the very southern end of the lake. This distribution points to a large migration wave that was overlaid by subsequent migration waves and masked by lineage sorting in most areas. The survival of this haplotype subgroup at both ends of the lake might point to a lesser chance to be overlaid at such tip-populations. The observed widespread but rare distribution pattern of the pink TCS-1 subcluster in fact supports our suggestion of at least two arrivals of TCS-1 haplotype fish in the very North of the Lake.

\section{Taxonomic considerations}

It is important to note that the distribution of mtDNA haplotypes, in combination with nuclear DNA markers, morphology and color, indicates considerable levels of past gene flow among entities considered as valid biological species. It was suggested that massive perturbations of the habitat or availability of new habitat cause species to interbreed even if they would not do so under stable conditions (Rüber et al., 2001; Seehausen, 2004). This scenario seems to fit many of the here studied Tropheus populations, as we found evidence for extensive mitochondrial introgression, which did not become evident in the nuclear data obtained so far.

Under particular circumstances hybridization upon secondary contact might actually lead to a novel 
evolutionary stable entity, as, for example, suggested for a number of animal taxa (Grant \& Grant, 2002; Gompert et al., 2006; Larsen et al., 2010; Sefc \& Koblmüller, 2016), including other cichlid fishes (Salzburger et al., 2002). It has been shown that hybrid populations can rapidly generate novel ("transgressive") phenotypes which might be rapidly sorted out via natural selection, or alternatively, that hybrid populations become isolated from their sources in particular habitats, to form a novel entity distinct from both parental species (Salzburger et al., 2002; Parsons et al., 2011). Also in Tropheus, the importance of hybridization for generating novel phenotypes has been proposed previously (Egger et al., 2007). Among the Tropheus morphs included here, Tropheus cf. brichardi 'Ubwari-green', exhibits an intermediate phenotype in form of greenish $T$. brichardi-like body color, blue eyes, but red-yellow $T$. sp. 'black' banding on the body flanks. In the mitochondrial data, all Tropheus cf. brichardi Ubwari-green samples analyzed group among $T$. sp. 'black,' whereas the AFLPbased nuclear DNA assignment also suggests intermediacy (Fig. 3). It is important to repeat here that this Tropheus lives in full sympatry with $T$. sp. 'black' Caramba at one particular shoreline at the western side of the Ubwari Peninsula between Cape Caramba and Muzimu (Brichard, 1978; Konings, 2013), so that speciation can be assumed as completed in these populations. The two species are not segregating spatially like many other sympatric Tropheus but fully coexist in similar abundancies (Ad Konings personal communication). As Tropheus cf. brichardi 'Ubwarigreen' combines features of both parental species and lives in sympatry with one of them, it clearly cannot be assigned to either of the described species, and should be regarded as a distinct species, for which hybrid origin seems likely.

Concerning taxonomic assignments, the populations from Ngombe to Muguruka should represent "pure" Tropheus brichardi, as these exclusively comprise TCS-1 haplotypes and because of phenotypic similarities to specimens collected in Nyanza Lac, the type locality. Unfortunately, no AFLP data are available for localities other than Nyanza Lac to support our species assignment also by nuclear multilocus data. All other populations except for $T$. cf. brichardi 'Ubwari-green' are pure $T$. sp. 'black.' Even if some populations show evidence for past mitochondrial introgression from $T$. brichardi and some morphological features considered atypical for T. sp. 'black' (Van Steenberge, 2014), our new AFLP data, in concordance with Egger et al. (2007), do not find evidence for large-scale genomic admixture in these introgressed populations. There is increasing evidence that in animals local or even range wide replacement of mitochondrial DNA, without signatures of nuclear genomic admixis, seems to be more common than previously thought (e.g., Nevado et al., 2009; Tang et al., 2012; Melo-Ferreira et al., 2014; Good et al., 2015; Koblmüller et al., 2016), such that taxonomic assignment based on mitochondrial data alone might be misleading.

Acknowledgments Open access funding provided by University of Graz. We wish to thank the members of the Centre de Recherche en Hydrobiologie at Uvira, Democratic Republic of the Congo, Prof. Gaspard Banyankimbona (University of Burundi), Maarten P. M. Vanhove, Radim Blazek, as well as Meirelle Schreyen and the staff of Fishes of Burundi, for their assistance during fieldwork. This study was supported by the Austrian Science Fund (grant P22737-B09 to CS). Field work of MVS was supported by the King Leopold III Funds for Nature Exploration and Conservation who, at the time, was recipient of a scholarship of the Research Foundation-Flanders (FWO Vlaanderen). Field work of SK was supported by the Czech Science Foundation (GBP505/12/ G112-ECIP).

Open Access This article is distributed under the terms of the Creative Commons Attribution 4.0 International License (http:// creativecommons.org/licenses/by/4.0/), which permits unrestricted use, distribution, and reproduction in any medium, provided you give appropriate credit to the original author(s) and the source, provide a link to the Creative Commons license, and indicate if changes were made.

\section{References}

Baric, S., W. Salzburger \& C. Sturmbauer, 2003. Phylogeography and evolution of the Tanganyikan cichlid genus Tropheus based upon mitochondrial DNA sequences. Journal of Molecular Evolution 56: 54-68.

Benjamini, Y. \& Y. Hochberg, 1995. Controlling the false discovery rate: a practical and powerful approach for multiple testing. Journal of the Royal Statistical Society, Series B 57: 289-300.

Brichard, P., 1978. Fishes of Lake Tanganyika. TFH Publications, Neptune City, USA.

Brown, R. P. \& Z. Yang, 2011. Rate variation and estimation of divergence times using strict and relaxed clocks. BMC Evolutionary Biology 11: 271.Clement, M., D. Posada \& K. A. Crandall, 2000. TCS: a computer program to estimate gene genalogies. Molecular Ecology 9: 1657-1659.

Cassanova, J. \& C. Hillaire-Marcel, 1992. Late Holocene hydrological history of Lake Tanganyika, East Africa, 
from isotopic data on fossil stromatolites. Palaeogeography, palaeoclimatology, Palaeoecology 91: 35-48.

Chikhi, L., V. C. Sousa, P. Luisi, B. Goossens \& M. A. Beaumont, 2010. The confounding effects of population structure, genetic diversity and the sampling scheme on the detection and quantification of population size changes. Genetics 186: 983-995.

Cohen, A. S., K. E. Lezzar, J. J. Tiercelin \& M. Soreghan, 1997. New palaeogeographic and lake-level reconstructions of Lake Tanganyika: implications for tectonic climatic and biological evolution in a rift lake. Basin Research 9: 107-132.

Cohen, A. S., J. R. Stone, K. R. M. Beuning, et al., 2007. Ecological consequences of early Late Pleistocene megadroughts in tropical Africa. Proceedings of the National Academy of Sciences of the United States of America 104: $16422-16427$.

Drummond, A. J. \& A. Rambaut, 2007. BEAST: Bayesian evolutionary analysis by sampling trees. BMC Evolutionary Biology 7: 214.

Drummond, A. J., A. Rambaut, B. Shapiro \& O. G. Pybus, 2005. Bayesian coalescent inference of past population dynamics from molecular sequences. Molecular Biology and Evolution 22: 1185-1192.

Duftner, N., S. Koblmüller \& C. Sturmbauer, 2005. Evolutionary relationships of the Limnochromini, a tribe of benthic deepwater cichlid fishes endemic to Lake Tanganyika. Journal of Molecular Evolution 60: 277-289.

Duftner, N., K. M. Sefc, S. Koblmüller, B. Nevado, E. Verheyen, H. Phiri \& C. Sturmbauer, 2006. Distinct population structure in a phenotypically homogeneous rock-dwelling cichlid fish from Lake Tanganyika. Molecular Ecology 15: 2381-2395.

Egger, B., S. Koblmüller, C. Sturmbauer \& K. M. Sefc, 2007. Nuclear and mitochondrial data reveal different evolutionary processes in the Lake Tanganyika cichlid genus Tropheus. BMC Evolutionary Biology 7: 137.

Excoffier, L. \& H. E. L. Lischer, 2010. Arlequin suite ver 3.5: a new series of programs to perform population genetics analyses under Linux and Windows. Molecular Ecology Resources 10: 564-567.

Excoffier, L., P. E. Smouse \& J. M. Quattro, 1992. Analysis of molecular variance inferred from metric distances among DNA haplotypes: application to human mitochondrial restriction data. Genetics 131: 479-491.

Genner, M. J., M. E. Knight, M. P. Haesler \& G. F. Turner, 2010. Establishment and expansion of Lake Malawi rock fish populations after a dramatic Late Pleistocene lake level rise. Molecular Ecology 19: 170-182.

Gompert, Z., J. A. Fordyce, M. L. Forister, A. M. Shaprio \& C. C. Nice, 2006. Homoploid hybrid speciation in an extreme habitat. Science 314: 1923-1925.

Good, J. M., D. Vanderpool, S. Keeble \& K. Bi, 2015. Neglible nuclear introgression despite complete mitochondrial capture between two species of chipmunks. Evolution 69: 1961-1972.

Grant, P. R. \& B. R. Grant, 2002. Enpredictable evolution in a 30-year study of Darwin's finches. Science 296: 707-711.

Hasegawa, M., H. Kishino \& T. Yano, 1985a. Dating of the human-ape splitting by a molecular clock of mitocondrial DNA. Journal of Molecular Evolution 21: 160-174.
Hasegawa, M., H. Kishino \& T.-A. Yano, 1985b. Dating of the human-ape splitting by a molecular clock of mitochondrial DNA. Journal of Molecular Evolution 22: 160-174.

Heller, R., L. Chikhi \& H. R. Siegismund, 2013. The confounding effect of population structure on Bayesian skyline plot inferences of demographic history. PLoS One 8: e62992.

King, R. B. \& R. Lawson, 1995. Color-pattern variation in Lake Erie water snakes: the role of gene flow. Evolution 49: 885-896.

Koblmüller, S., K. M. Sefc, N. Duftner, M. Warum \& C. Sturmbauer, 2007. Genetic population structure as indirect measure of dispersal ability in a Lake Tanganyika cichlid. Genetica 130: 121-131.

Koblmüller, S., N. Duftner, K. M. Sefc, U. Aigner, M. Rogetzer \& C. Sturmbauer, 2009. Phylogeographic structure and gene flow in the scale-eating cichlid Perissodus microlepis (Teleostei, Perciformes, Cichlidae) in southern Lake Tanganyika. Zoologica Scripta 38: 257-268.

Koblmüller, S., B. Egger, C. Sturmbauer \& K. M. Sefc, 2010. Rapid radiation, ancient incomplete lineage sorting and ancient hybridization in the endemic Lake Tanganyika cichlid tribe Tropheini. Molecular Phylogenetics and Evolution 55: 318-334.

Koblmüller, S., W. Salzburger, B. Obermüller, E. Eigner, C. Sturmbauer \& K. M. Sefc, 2011. Separated by sand, fused by dropping water: habitat barriers and fluctuating water levels steer the evolution of rock-dwelling cichlid populations in Lake Tanganyika. Molecular Ecology 20: 2272-2290.

Koblmüller, S., B. Nevado, L. Makasa, M. Van Steenberge, M. P. M. Vanhove, E. Verheyen, C. Sturmbauer \& K. M. Sefc, 2016. Phylogeny and phylogeography of Altolamprologus: ancient introgression and recent divergence in a rockdwelling Lake Tanganyika cichlid genus. Hydrobiologia. doi:10.1007/s10750-016-2896-2.

Konings, A., 2013. Tropheus in their natural habitat. Cichlid Press, El Paso. 160 pp.

Kuhner, M. K., 2009. Coalescent genealogy samples: windows into population history. Trends in Ecology and Evolution 24: 86-93.

Larsen, P. A., M. R. Marchán-Rivadeneira \& R. J. Baker, 2010. Natural hybridization generates mammalian lineage with species characteristics. Proceedings of the National Academy of Sciences USA 107: 11447-11452.

Lee, W.-J., J. Conroy, W. H. Howell \& T. D. Kocher, 1995. Structure and evolution of the teleost mitochondrial control region. Journal of Molecular Evolution 41: 54-66.

Leigh, J. W. \& D. Bryant, 2015. POPART: full-feature software for haplotype network construction. Methods in Ecology and Evolution 6: 1110-1116.

Lezzar, K. E., J. J. Tiercelin, M. De Batist, A. S. Cohen, T. Bandora, P. Van Rensbergen, C. Le Turdu, W. Mifundu \& J. Klerkx, 1996. New seismic stratigraphy and Late Tertiary history of the North Tanganyika Basin, East African Rift system, deduced from multichannel and high-resolution reflections seismic data and piston core evidence. Basin Research 8: 1-28.

Lezzar, K. E., J. J. Tiercelin, C. Le Turdu, A. S. Cohen, D. J. Reynolds, B. Le Gall \& C. A. Scholz, 2002. Control of normal fault interaction on the distribution of major 
Neogene sedimentary depocenters, Lake Tanganyika, East African rift. The American Association of Petroleum Geologists 6: 1027-1059.

Mattersdorfer, K., S. Koblmüller \& K. M. Sefc, 2012. AFLP genome scans suggest divergent selection on colour patterning in allopatric colour morphs of a cichlid fish. Molecular Ecology 21: 3531-3544.

McGlue, M. M., K.-E. Lezzar, A. S. Cohen, J. M. Russell, J.-J. Tiercelin, A. A. Felton, E. Mbede \& H. H. Nkotagu, 2008. Seismic records of late Pleistocene aridity in Lake Tanganyika, tropical East Africa. Journal of Paleolimnology 40: 635-653.

Melo-Ferreira, J., F. A. Seixas, E. Cheng, L. S. Mills \& P. C. Alves, 2014. The hidden history of the snowshoe hare, Lepus americanus: extensive mitochondrial DNA introgression inferred from multilocus genetic variation. Molecular Ecology 18: 4617-4630.Meyer, A., J. M. Morrissey \& M. Schartl, 1994. Recurrent origin of sexually selected trait in Xiphophorus fishes inferred from a molecular phylogeny. Nature 368: 539-541.

Nei, M. \& W.-H. Li, 1979. Mathematical model for studying genetic variation in terms of restriction endonucleases. Proceedings of the National Academy of Sciences USA 76: 5269-5273.

Nevado, B., S. Koblmüller, C. Sturmbauer, J. Snoeks, J. UsanoAlemany \& E. Verheyen, 2009. Complete mitochondrial DNA replacement in a Lake Tanganyika cichlid fish. Molecular Ecology 18: 4240-4255.

Nevado, B., S. Mautner, C. Sturmbauer \& E. Verheyen, 2013. Water-level fluctuations and metapopulation dynamics as drivers of genetic diversity of three Tanganyikan cichlid fish species. Molecular Ecology 22: 3933-9348.

Nolte, A. W. \& D. Tautz, 2010. Understanding the onset of hybrid speciation. Trends in Genetics 26: 54-58.

Parsons, K. J., H. S. Young \& R. C. Albertson, 2011. Hybridization promotes evolvability in African cichlids: connections between transgressive segregation and phenotypic integration. Evolutionary Biology 38: 306-315.

Pflumio, C., J. Boulègue \& J.-J. Tiercelin,1994. Hydrothermal activity in the Northern Tanganyika Rift, East Africa. Chemical Geology 116: 85-109.

Pinho, C. \& J. Hey, 2010. Divergence with gene flow: models and data. Annual Review of Ecology, Evolution, and Systematics 41: 215-240.

Poll, M., 1986. Classification des Cichlidae du lacTanganika. tribus, genres et espèces. Académie Royale de Belgique, Memoires de la Classe des Sciences 45: 1-163.Posada, D., 2008. jModelTest: phylogenetic model averaging. Molecular Biology and Evolution 25: 1253-1256.

$\mathrm{R}$ core team, 2015. R: A language and environment for statistical computing, R foundation for statistical computing, Vienna, Austria. https://www.R-project.org

Richlen, M. L. \& P. H. Barber, 2005. A technique for the rapid extraction of microalgal DNA from single live and preserved cells. Molecular Ecology Notes 5: 688-691.

Rossiter, A., 1995. The cichlid fish assemblages of Lake Tanganyika: ecology, behaviour and evolution of its species flocks. In: Begon, M. \& A. H. Fitter (eds). Advances in Ecological Research. Academic Press, New York: 187-252.
Rozas, J., P. Librado, J. C. Sánchez-DelBarrio, X. Messeguer \& R. Rozas, 2010. DnaSP5.10, Universitat de Barcelona

Rüber, L., E. Verheyen \& A. Meyer, 1999. Replicated evolution of trophic specializations in an endemic cichlid fish lineage from Lake Tanganyika. Proceedings of the National Academy of Sciences USA 96: 10230-10235.

Rüber, L., A. Meyer, C. Sturmbauer \& E. Verheyen, 2001. Population structure in two sympatric species of the Lake Tanganyika cichlid tribe Eretmodini: evidence for introgression. Molecular Ecology 10: 1207-1225.

Salzburger, W., S. Baric \& C. Sturmbauer, 2002. Speciation via introgressive hybridization in East African cichlids? Molecular Ecology 11: 619-625.

Schneider, K., S. Koblmüller \& K. M. Sefc, 2016. HExT, a software supporting tree-based screens for hybrid taxa in multilocus data sets, and an evaluation of the homoplasy excess test. Methods in Ecology and Evolution 7: 358-368.

Scholz, C. A., J. W. King, G. S. Ellis, P. K. Swart, J. C. Stager \& S. M. Colman, 2003. Paleolimnology of Lake Tanganyika, East Africa, over the past $100 \mathrm{k}$ yr. Journal of Paleolimnology 30: 139-150.

Schupke, P., 2003. Cichlids of Lake Tanganyika, Part 1: The species of the genus Tropheus, Aqualog, Rodgau, Germany: $190 \mathrm{pp}$.

Seehausen, O., 2004. Hybridization and adaptive radiation. Trends in Ecology and Evolution 19: 198-207.

Sefc, K. M. \& S. Koblmüller, 2016. Ancient hybrid origin of the eastern wolf not yet off the table: a comment on Rutledge et al. (2015). Biology Letters 12: 20150834.

Sefc, K. M., S. Baric, W. Salzburger \& C. Sturmbauer, 2007. Species-specific population structure in rock-specialized sympatric cichlid species in Lake Tanganyika, East Africa. Journal of Molecular Evolution 64: 33-49.

Sefc, K. M., K. Mattersdorfer, C. M. Hermann \& S. Koblmüller, 2016. Past lake shore dynamics explain present pattern of unidirectional introgression across a habitat barrier. Hydrobiologia. doi:10.1007/s10750-016-2791-x.

Sturmbauer, C. \& A. Meyer, 1992. Genetic divergence, speciation, and morphological stasis in a lineage of African cichlid fishes. Nature 358: 578-581.

Sturmbauer, C., E. Verheyen, L. Rüber \& A. Meyer, 1997. Phylogeographic patterns in populations of cichlid fishes from rocky habitats in Lake Tanganyika. In Kocher, T. D. \& C. Stepien (eds), Molecular Phylogeny of Fishes. Academic Press, New York: 97-111.

Sturmbauer, C., S. Koblmüller, K. Sefc \& N. Duftner, 2005. Phylogeographic history of the genus Tropheus, a lineage of rock-dwelling cichlid fishes endemic to Lake Tanganyika. Hydrobiologia 542: 335-366.

Sturmbauer, C., M. Husemann \& P. Danley, 2011. Explosive speciation and adaptive radiation of East African cichlid fishes. In: Zachos, F.E. \& J.C. Habel (eds), Biodiversity hotspots - distribution and protection of conservation priority areas. Springer, Berlin: 333-362.

Tamura, K. \& M. Nei, 1993. Estimation of the number of nucleotide substitutions in the control region of mitochondrial DNA in humans and chimpanzees. Molecular Biology and Evolution 10: 512-526.

Tamura, K., G. Stecher, D. Peterson, A. Filipski \& S. Kumar, 2013. MEGA6: Molecular Evolutionary Genetics Analysis 
Version 6.0. Molecular Biology and Evolution 30: 2725-2729.

Tang, Q.-Y., S.-Q. Liu, D. Yu, H.-Z. Liu \& P. D. Danley, 2012. Mitochondrial capture and incomplete lineage sorting in the diversification of balitorine loaches (Cypriniformes, Balitoridae) revealed by mitochondrial and nuclear genes. Zoologica Scripta 41: 233-247.

Templeton, A. R., K. A. Crandall \& C. F. Sing, 1992. A cladistic analysis of phenotypic associations with haplotypes inferred from restriction endonuclease mapping and DNA sequence data. III. Cladogram estimation. Genetics 132: 619-633.

Van Steenberge, M., 2014. Species and speciation in Tropheus, Simochromis and Pseudosimochromis - a multidisciplinary approach to a cichlid radiation from Lake Tanganyika. PhD Thesis, Univ. Leuven, Belgium. ISBN: 978-90-8649-754-6. 341 pp.

Van Steenberge, M., M. P. M. Vanhove, D. Muzumani Risasi, T. Mulimbwa N'simbula, F. Muterezi Bukinga, A. Pariselle, C. Gillardin, E. Vreven, J. A. M. Raeymaekers, T. Huyse, F. A. M. Volckaert, V. Nshombo Muderhwa, J. Snoeks, 2011. A recent inventory of the fishes of the north-western and central western coast of Lake Tanganyika (Democratic
Republic Congo). Acta Ichthyologica et Piscatoria 41: 201-214. doi:10.3750/AIP2011.41.3.08

Van Steenberge, M., M. P. N. Vanhove, F. C. Breman \& J. Snoeks, 2015. Complex geographical variation patterns in Tropheus duboisi Marlier, 1959 (Perciformes, Cichlidae) from Lake Tanganyika. Hydrobiologia 748: 39-60. doi:10. 1007/s10750-013-1692-5.

Verheyen, E., L. Rüber, J. Snoeks \& A. Meyer, 1996. Mitochondrial phylogeny of rock-dwelling cichlid fishes reveals evolutionary influence of historical lake level fluctuations of Lake Tanganyika, Africa. Philosophical transactions of the Royal Society London B 351: 797-805.

Weir, B. S. \& C. C. Cockerham, 1984. Estimating F-statistics for the analysis of population structure. Evolution 38: 1358-1370.

Whitlock, R., H. Hipperson, M. Mannarelli, R. K. Butlin \& T. Burke, 2008. An objective, rapid and reproducible method for scoring AFLP peak-height data that minimizes genotyping error. Molecular Ecology Resources 8: 725-735.

Winkelmann, K., L. Rüber \& M. J. Genner, 2016. Lake level fluctuations and divergence of cichlid fish ecomorphs in Lake Tanganyika. Hydrobiologia. doi:10.1007/s10750016-2839-y. 